\title{
Model for Identifying High Carotid Body Chemosensitivity in Patients with Obstructive Sleep Apnea
}

\author{
Hong Peng $\mathrm{Li}^{1,2, *}$ \\ Hai Qin Wang ${ }^{3, *}$ \\ Ning $\mathrm{Li}^{1,2, *}$ \\ Liu Zhang $\mathbb{D}^{1,2}$ \\ Shi Qi Li (iD ${ }^{1,2}$ \\ Ya Ru Yan ${ }^{1,2}$ \\ Huan Huan Lu',2 \\ Yi Wang ${ }^{1,2}$ \\ Xian Wen Sun ${ }^{1,2}$ \\ Ying Ni Lin ${ }^{1,2}$ \\ Jian Ping Zhou ${ }^{1,2}$ \\ Qing Yun $\mathrm{Li}^{1,2}$
}

'Department of Respiratory and Critical Care Medicine, Ruijin Hospital, Shanghai jiao Tong University School of Medicine, Shanghai, 200025, People's Republic of China; ${ }^{2}$ Institute of Respiratory Medicine, Shanghai Jiao Tong University School of Medicine, Shanghai, 200025, People's Republic of China; ${ }^{3}$ Xietu Community Health Service Center of Xuhui District, Shanghai, 20023I, People's Republic of China

*These authors contributed equally to this work
Correspondence: Qing Yun Li

Department of Respiratory and Critical Care Medicine, Ruijin Hospital, Shanghai Jiao Tong University School of Medicine, No. 197 Rui Jin 2nd Road, Shanghai 200025, PR China; Institute of Respiratory Medicine, Shanghai Jiao Tong University School of Medicine, No. 197

Rui Jin 2nd Road, Shanghai, 200025,

People's Republic of China

Tel +86-2I-64370045

Fax +86-2I-62836805

Email liqingyun68@hotmail.com
Objective: The carotid body $(\mathrm{CB})$ is a major peripheral respiratory chemoreceptor. In patients with obstructive sleep apnea (OSA), high CB chemosensitivity (CBC) is associated with refractory hypertension and insulin resistance and known to further aggravate OSA. Thus, the identification of high $\mathrm{CB}$ ( $\mathrm{hCBC}$ ) among OSA patients is of clinical significance, but detection methods are still limited. Therefore, this study aimed to explore the association of $\mathrm{CBC}$ with OSA severity and to develop a simplified model that can identify patients with hCBC.

Methods: In this cross-sectional study of subjects who underwent polysomnography (PSG), $\mathrm{CBC}$ was measured using the Dejours test. We defined hCBC as a decrease of $>12 \%$ in respiratory rate (RR) after breathing of pure $\mathrm{O}_{2}$. The association of CBC with OSA severity was explored by logistic regression, and a model for identifying hCBC was constructed and confirmed using receiver operating characteristic analysis.

Results: Patients with OSA ( $\mathrm{n}=142)$ and individuals without OSA $(\mathrm{n}=38)$ were enrolled. CBC was higher in patients with OSA than in those without OSA (\% decrease in RR, $15.2 \% \pm 13.3 \%$ vs $9.1 \% \pm 7.5 \%, \mathrm{P}<0.05$ ). Apnea-hypopnea index (AHI), fraction of apnea-hypopnea events in rapid-eye-movement sleep ( $\left.\mathrm{F}_{\text {events-in-REM}}\right)$, and longest time of apnea (LTA) were associated with $\mathrm{hCBC}$ independently (odds ratio $[\mathrm{OR}]=1.048, \mathrm{OR}=1.082$, and $\mathrm{OR}=1.024$ respectively; all $P<0.05$ ). The model for identifying hCBC allocated a score to each criterion according to its OR values, ie, 1 (LTA $>48.4 \mathrm{~s}$ ), 2 (AHI $>15.7$ events/hour), and 3 ( $\mathrm{F}_{\text {events-in-REM }}>12.7 \%$ ). A score of 3 or greater indicated hCBC with a sensitivity of $79.4 \%$ and specificity of $88.2 \%$. Conclusion: High CBC is associated with the severity of OSA. A simplified scoring system based on clinical variables from PSG can be used to identify hCBC.

Keywords: carotid body, chemosensitivity, obstructive sleep apnea, severity, indicator

\section{Introduction}

Obstructive sleep apnea (OSA) is a heterogeneous and increasingly common disorder characterized by repetitive narrowing and collapse of the pharyngeal airway during sleep. ${ }^{1}$ The main feature of OSA is chronic intermittent hypoxia $(\mathrm{CIH})$, which is associated with conditions such as hypertension, metabolic disorders, and cancer. ${ }^{2-4}$ The pathogenesis of OSA still remains incompletely understood, but an unstable respiratory control system is one of the major non-anatomical mechanisms. ${ }^{5}$ The respiratory control instability in OSA can be quantified using the gain of the ventilatory control feedback loop (loop gain, LG). The LG is elevated in OSA, and lowering the LG with acetazolamide or oxygen inhalation results in a reduction in OSA severity. ${ }^{6-8}$ 
The carotid body $(\mathrm{CB})$ is a key peripheral respiratory chemoreceptor that sense changes in the partial pressures of $\mathrm{O}_{2}$ and $\mathrm{CO}_{2}$ as well as the $\mathrm{pH}$ level in arterial blood and induce a ventilation response. High $\mathrm{CB}$ chemosensitivity $(\mathrm{hCBC})$ is defined by an elevated ventilatory response to hypoxia or hypercapnia. ${ }^{9}$ The $\mathrm{CB}$ enhances the sensitivity of central chemoreceptors to hypercapnia through afferent innervation. $^{10}$ In addition, hyperoxia induces a "physiological denervation" of the CB that may inhibit the normal ventilation responsiveness. ${ }^{11}$ Thus, CBC is a principal component of the ventilatory control feedback loop, based on the dual role of the $\mathrm{CB}$ in the regulation of both peripheral and central chemosensitivity to the partial pressures of $\mathrm{O}_{2}$ and $\mathrm{CO}_{2} .{ }^{12}$ Thus, hCBC is related to respiratory control instability, which is one of the major pathogenic traits of OSA. ${ }^{5}$

Additionally, OSA patients exhibit enhanced cardiovascular reflex responses to acute hypoxia, and this is attributed to potentiation of $\mathrm{CB}$ activity. ${ }^{13,14}$ Previous research has demonstrated associations between hCBC and refractory hypertension and insulin resistance. ${ }^{15}$ Therefore, it is necessary to identify hCBC for effective management of OSA patients. Notably, the relationship between increased CB activity and OSA severity has not been characterized. Additionally, the gold standard method for determining $\mathrm{CBC}$ is the double-breath Dejours test, ${ }^{16,17}$ which is relatively inconvenient in clinical practice. In the present study, we hypothesized that $\mathrm{CBC}$ is associated with OSA severity, and thus, it is possible to develop a simplified assessment model for identifying hCBC using clinical parameters from polysomnography (PSG). To test these hypotheses, we explored the association of CBC with OSA severity and evaluated the effectiveness of a model based on PSG parameters as a clinical tool for identifying hCBC.

\section{Methods}

\section{Ethical Approval}

This study was approved by the ethics committee of Ruijin Hospital ([2018] No. 107). Furthermore, it was performed in accordance with the principles of the Declaration of Helsinki. Written informed consent was obtained from all participants.

\section{Participants}

For this prospective cross-sectional study, all participants were recruited before they underwent PSG at the Sleep Disorders
Center of Ruijin Hospital Affiliated to Shanghai Jiao Tong University School of Medicine from June 2020 to September 2020. The inclusion criteria were snoring, daytime sleepiness (Epworth Sleepiness Scale, ESS >6), refractory hypertension (failure of at least three antihypertensive drugs, including diuretics, to control systolic blood pressure below $140 \mathrm{mmHg}$ and/or diastolic blood pressure below $90 \mathrm{mmHg}$ ), and sudden awakening due to gasping for breath. ${ }^{18}$ The exclusion criteria were age $<18$ or $>80$ years, pregnancy, sleep disorders other than OSA (eg, insomnia, or narcolepsy), previous continuous positive airway pressure (CPAP) treatment for OSA, long-term sojourn in high altitude areas, chronic respiratory failure $\left(\mathrm{PaO}_{2}<60 \mathrm{mmHg}\right.$ or diagnosed by medical history), long-term home oxygen therapy, and cardiac insufficiency (New York Heart Association [NYHA) II-IV).

Volunteers for health examination in Ruijin Hospital with a healthy phenotype were collected to validate the results of Dejours test and the normal range of $\mathrm{CBC}$.

\section{Study Design}

A flowchart of the study participants is presented in Figure 1. All participants completed the ESS upon enrollment. The sociodemographic and anthropometric data and comorbidities were documented. Height, weight, neck circumference, and abdominal circumference were assessed using standardized protocols. Body mass index (BMI) was calculated as the body weight $(\mathrm{kg})$ divided by the body height squared $\left(\mathrm{m}^{2}\right)$. Blood pressure was measured, and hypertension was defined as a systolic blood pressure $\geq 140 \mathrm{mmHg}$, diastolic blood pressure $\geq 90 \mathrm{mmHg}$, or a clinical diagnosis of hypertension and current prescription of antihypertensive medication. ${ }^{19}$ Smokers were those who regularly had smoked 10-20 cigarettes/day for at least 3 years. ${ }^{20}$

\section{PSG Monitoring}

All participants underwent an in-laboratory PSG examination (Alice 6, Philips Respironics, PA, USA). They were asked to refrain from alcohol, caffeine, sedatives, or hypnotics on the test day. The PSG channels included four electroencephalograms, a submental electromyogram, two electrooculograms (right and left), two electrocardiography channels, pulse oxygen saturation, oral and nasal airflow, nasal air pressure, thoracic-abdominal respiratory movement, snoring microphone, and body position. Only edited records with more than $4 \mathrm{~h}$ of good-quality signals were included. The apnea-hypopnea index (AHI) referred to the total event numbers of apnea and hypopnea per hour of sleep. Apnea was defined by a respiratory event with 


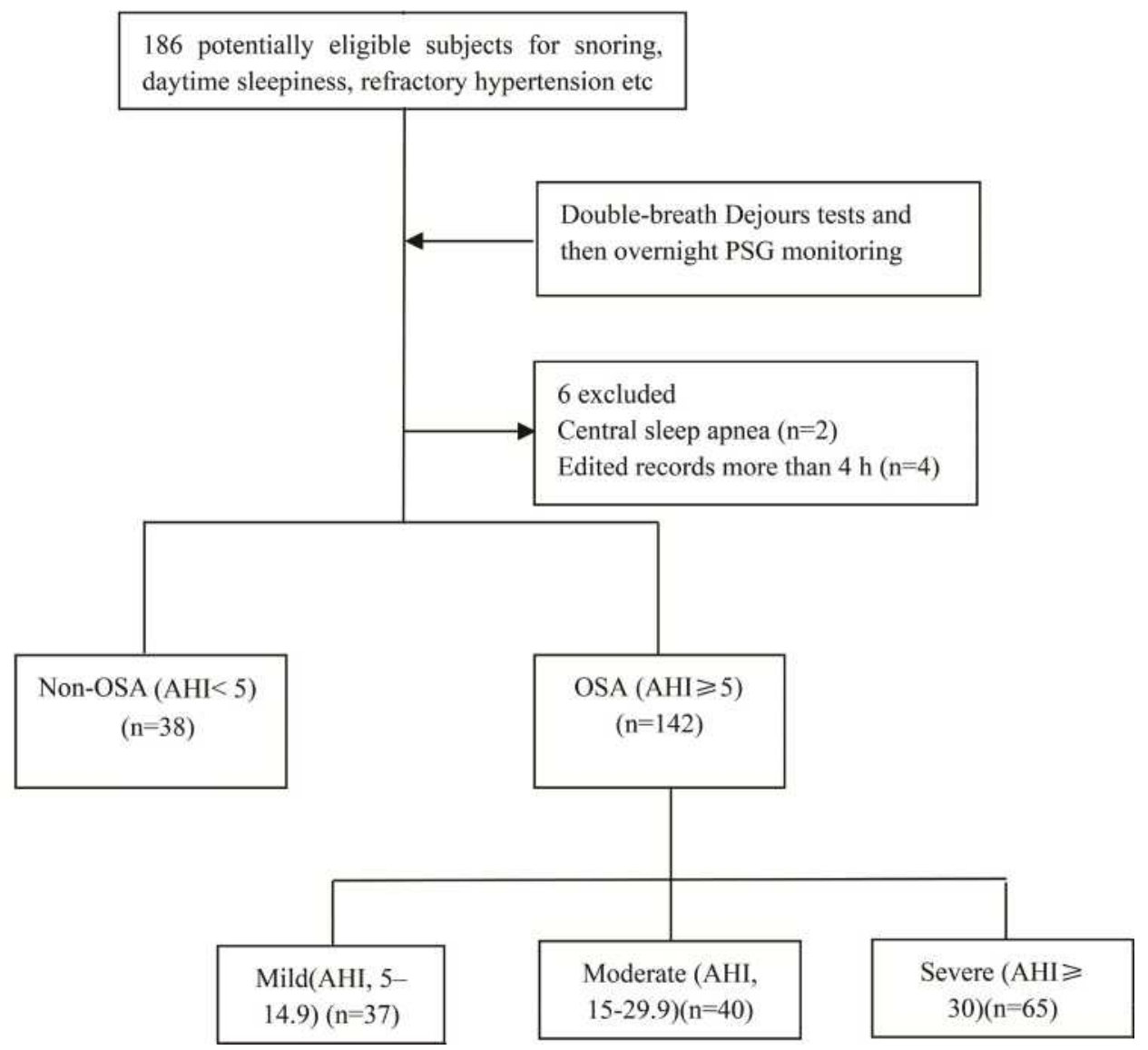

Figure I Flowchart of the study participants.

a drop in nasal airflow by $\geq 90 \%$, and hypopnea was defined by a drop in peak signal excursions by $\geq 30 \%$ of pre-event baseline using nasal pressure for at least 10 seconds with $a \geq 4 \%$ oxygen desaturation from pre-event baseline. The event was considered OSA if there was effort to breath during apnea or hypopnea. The event was considered central sleep apnea (CSA) if there was no effort to breath during apnea or hypopnea. ${ }^{18}$ The oxygen desaturation index (ODI) was defined as the total number of at least $4 \%$ oxygen desaturations per hour of sleep. The mean pulse oxygen saturation $\left(\mathrm{SpO}_{2}\right)$ was defined as the mean oxygen saturation during the sleep period. The nadir $\mathrm{SpO}_{2}$ was the lowest oxygen saturation during apnea or hypopnea periods. The percentage of sleep duration with $\mathrm{SpO}_{2}$ $<90 \%$ (TS90) was determined. OSA was defined as mild (AHI, 5-14 events per hour), moderate (15-29 events per hour), or severe ( $>30$ events per hour). Based on the AHI, the participants were categorized into two groups: the non-OSA group (AHI $<5$ events per hour) and the OSA group (AHI $\geq 5$ events per hour).

\section{CBC Detected by Dejours Test}

$\mathrm{CBC}$ was determined by the Dejours test (double-breath $\mathrm{O}_{2}$ test), ${ }^{16,17}$ as the $\mathrm{CB}$ chemoreflex sensitivity to hypoxia (mainly) and hypercapnia. During the Dejours test, the participants breathed room air first, then two breaths of pure $\mathrm{O}_{2}$ delivered at a $10 \mathrm{~L} / \mathrm{min}$ flow, and then room air again, via a mouthpiece connected to a three-way valve in a pressure-type spirometer system (TOW-Intelligent Technology, Shanghai, China). The curve of respiratory flow was recorded, and the respiratory rate (RR) and the tidal volume (VT) were calculated by the spirometer system. In the Dejours test, the inhalation of two tidal volumes of pure $\mathrm{O}_{2}$ results in a decrease in ventilation ( $\mathrm{RR}$ or VT) that reflects $\mathrm{CBC}$ and can be expressed as the percentage of decrease of the pre-oxygen RR or VT. ${ }^{16,17}$ Because the transient fall in ventilation occurs after a short lag and is maximal at 10-20 s after the $\mathrm{O}_{2}$ inhalation in resting subjects, ${ }^{17}$ the $\%$ decrease in RR or VT was calculated as follows: (the mean RR or VT values during the $20 \mathrm{~s}$ after pure oxygen breathing minus the mean of RR or VT during room air breathing) divided by the mean of RR or VT during 
room air breathing (Figure 2A). The test was repeated three times for each participant to assess reproducibility.

\section{Statistical Analysis}

All statistical analyses were performed using SPSS 20.0 for Windows (IBM Corp., Armonk, NY, USA). $P$-values $<0.05$ were considered statistically significant. Continuous variables are expressed as mean \pm standard deviation (SD). Normal distribution was assessed using the Kolmogorov-Smirnov test. According to the distribution, one-way analysis of variance (ANOVA) or the Kruskal-Wallis test was used for multiple comparisons among groups. Categorical variables are presented as a percentage and were analyzed using Fisher's exact test. Pearson's correlation analysis was used to investigate the correlation between clinical characteristics, PSG data, and $\mathrm{CBC}$. Multivariable stepwise linear regression analysis was performed to study the associations between the defined independent variables and $\mathrm{CBC}$ (as the dependent variable). Multivariable regression was used to determine the independent indicators for hCBC. Receiver operating characteristic (ROC) curves were generated for each independent indicator to derive the best cutoff values. Dichotomized variables according to those cutoffs were then used in a multivariable logistic regression model to identify the independent indicators that could be included in a clinical scoring tool and determine the weighting they could be given according to their odds ratio (OR) values. The sensitivity and specificity of this scoring tool were determined by ROC curve analysis.

\section{Results}

\section{Characteristics of Study Participants}

The demographic characteristics and PSG data for the study participants are presented in Table 1 and Supplementary Table 1. The 180 participants recruited for this study had a mean age of $44.8 \pm 12.9$ years, a mean BMI of $25.6 \pm 4.2 \mathrm{~kg} / \mathrm{m}^{2}$, and a mean AHI of $27.7 \pm 9.3$ events per hour. Higher values of age, BMI, ESS score, AHI, TS90, longest time of apnea
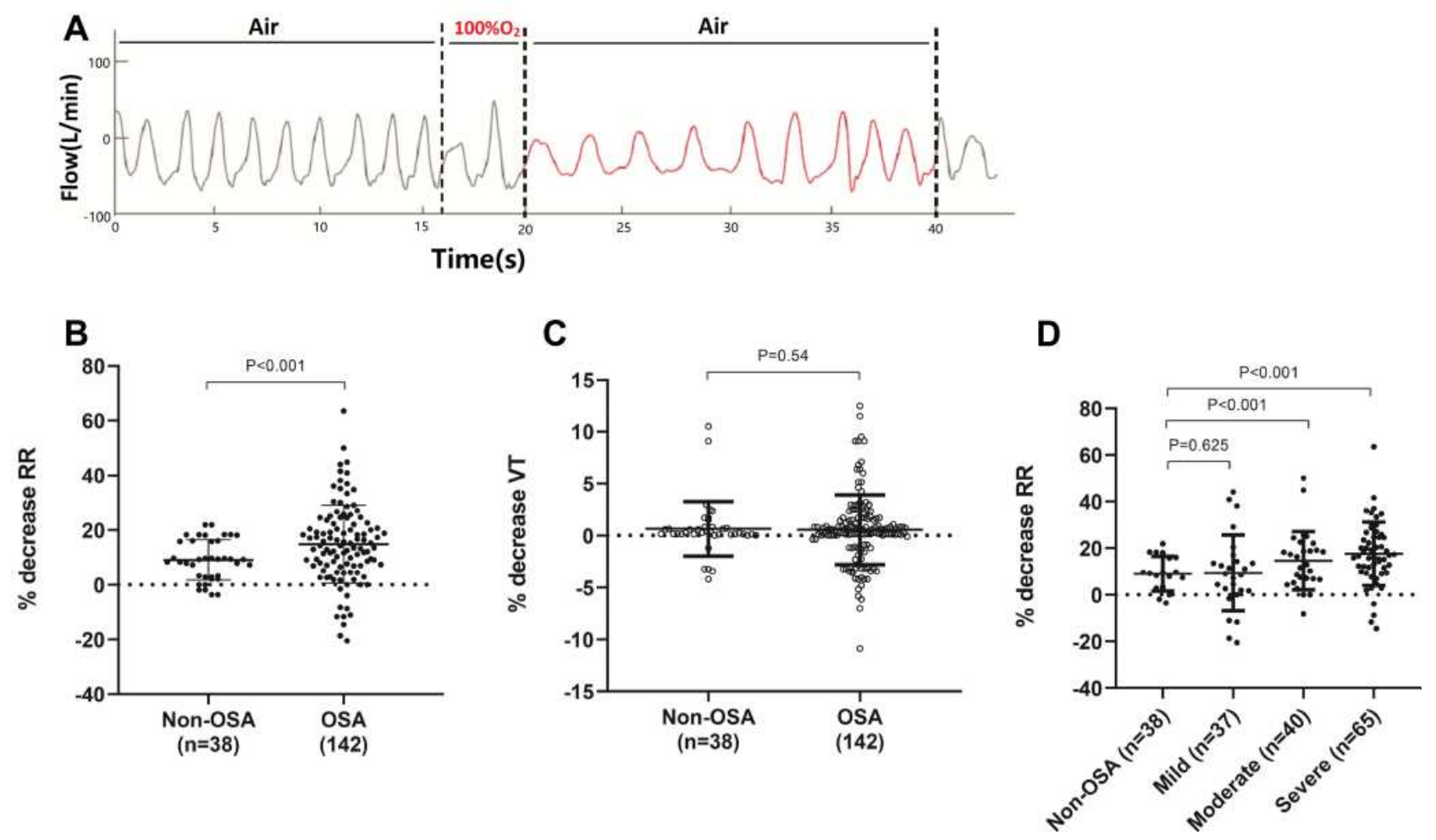

Figure 2 Carotid body chemosensitivity (CBC) in non-OSA individuals and OSA patients. (A) Respiratory flow curve from pressure-type spirometer for the effect of I00\% $\mathrm{O}_{2}$ (hyperoxia) on spontaneous ventilation. (B and $\mathbf{C}$ ) Comparison of Dejours test results between the non-OSA and OSA groups. CBC was evaluated as the \% decrease in respiratory rate (RR) or tidal volume (VT) produced by two breaths of $100 \% \mathrm{O}_{2}$. The \% decrease in RR in the OSA group was significantly higher than that in the non-OSA group $(15.2 \% \pm 13.3 \%$ vs $9.1 \% \pm 7.5 \%, P<0.05)$, whereas the \% decrease in VT did not differ between the groups $(P=0.54)$. (D) Comparison of Dejours test results between the non-OSA group and the OSA severity subgroups. The moderate and severe OSA groups had significantly increased CBC compared with the non-OSA group based on the \% decrease in RR ( $14.6 \% \pm 12.5 \%$ and $17.6 \% \pm 13.6 \%$, respectively, vs $9.1 \% \pm 7.5 \%, P<0.05)$, and no difference was observed between the mild OSA and non-OSA groups. The \% decreases in RR and VT were calculated as follows: decrease in RR or VT (the mean RR or VT value during the period of $20 \mathrm{~s}$ after pure oxygen breathing minus the mean RR or VT during exposure to air) divided by the mean RR or VT during exposure to air. The red line shows the period of time used to calculate the mean RR and VT after oxygen inhalation. Values are shown as mean $\pm \mathrm{SD}$. 
Table I Demographic, Polysomnographic, and Ventilation Data from Dejours Test for the Non-OSA and OSA Groups

\begin{tabular}{|c|c|c|c|c|}
\hline Characteristics & All $(n=180)$ & Non-OSA Group $(n=38)$ & OSA Group $(n=\mid 42)$ & $P^{*}$ \\
\hline Age, years & $44.8 \pm 12.9$ & $40.1 \pm 12.9$ & $46.4 \pm 12.6$ & $<0.001$ \\
\hline Male, n (\%) & I36 (7I.6) & $34(70.8)$ & $102(7 \mid .8)$ & $<0.001$ \\
\hline BMI, $\mathrm{kg} / \mathrm{m}^{2}$ & $25.6 \pm 4.3$ & $24.1 \pm 3.8$ & $26.0 \pm 4.2$ & $<0.001$ \\
\hline ESS score & $4.8 \pm 1.4$ & $2.3 \pm 1.6$ & $5.2 \pm 1.3$ & $<0.001$ \\
\hline $\mathrm{AHI}$, events per hour & $27.7 \pm 9.3$ & $3.6 \pm 1.1$ & $30.4 \pm 12.0$ & $<0.001$ \\
\hline Mean $\mathrm{SpO}_{2}, \%$ & $94.5 \pm 2.8$ & $95.8 \pm 1.3$ & $93.7 \pm 3.1$ & $<0.001$ \\
\hline Nadir $\mathrm{SpO}_{2}, \%$ & $81.2 \pm 11.6$ & $89.6 \pm 4.3$ & $78.2 \pm 11.9$ & $<0.001$ \\
\hline TS90, \% & $8.7 \pm 4.5$ & $0.7 \pm 0.6$ & $13.2 \pm 3.8$ & $<0.001$ \\
\hline MAI, events per hour & $25.8 \pm 9.9$ & $|6| \pm 8.7$. & $31.3 \pm 10.4$ & $<0.001$ \\
\hline ODI, events per hour & $25.0 \pm 10.2$ & $1.7 \pm 0.2$ & $26.4 \pm 10.1$ & $<0.001$ \\
\hline LTA, min & $55.4 \pm 17.9$ & $22.3 \pm 7.47$ & $67.6 \pm 13.4$ & $<0.001$ \\
\hline $\mathrm{F}_{\text {events-in-REM, }}(\%)$ & $14.5 \pm 5.8$ & $10.5 \pm 3.5$ & $18.5 \pm 4.6$ & $<0.001$ \\
\hline Decrease in RR, \% & $14.8 \pm 9.9$ & $9.1 \pm 7.5$ & $15.2 \pm 13.3$ & $<0.001$ \\
\hline Decrease in $R R \geq 12 \%, n(\%)$ & $102(56.7)$ & $8(21.1)$ & $94(66.2)$ & $<0.001$ \\
\hline Decrease in VT, \% & $1.87 \pm 2.36$ & $1.84 \pm 2.23$ & $1.91 \pm 2.61$ & 0.54 \\
\hline
\end{tabular}

Note: $* P<0.05$ versus non-OSA group.

Abbreviations: BMI, body mass index; ESS, Epworth Sleepiness Scale; AHI, apnea-hypopnea index; $\mathrm{SpO}_{2}$, pulse oxygen saturation; TS90, percentage of sleep duration with

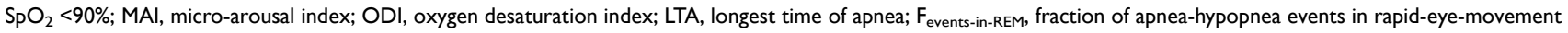
sleep (REM) (\% total events).

(LTA), and fraction of apnea-hypopnea events in rapid-eyemovement sleep ( $\%$ total events) ( $\left.F_{\text {events-in-REM }}\right)$ were observed in the OSA group compared with the non-OSA group (all $P<0.001$ ). The mean $\mathrm{SpO}_{2}$ and nadir $\mathrm{SpO}_{2}$ were significantly lower in the OSA group than in the non-OSA group (both $P<0.001$ ). The prevalence of hypertension $(27.7 \%)$ in the severe OSA subgroup was higher than in the non-OSA group (23.7\%) and the other OSA subgroups (10.8\% for the mild subgroup and $7.5 \%$ for the moderate subgroup) (Supplementary Table 1). To evaluate the effectiveness of Dejours test utilizing a spirometer system, an additional 35 healthy volunteers were recruited (Supplementary Table 4).

\section{Comparison of CBC Between the OSA and Non-OSA Groups}

$\mathrm{CBC}$ was significantly higher in OSA patients than in participants without OSA based on the \% decrease in RR $(15.2 \%$ $\pm 13.3 \%$ vs $9.1 \% \pm 7.5 \%,(P<0.05$; Figure $2 \mathrm{~B}$ and Table 1$)$. No significant differences were observed in the $\%$ decrease in VT between the groups $(P=0.54$; Figure $2 \mathrm{~B})$. Furthermore, patients with moderate and severe OSA showed significantly increased $\mathrm{CBC}$ compared with the non-OSA participants based on the $\%$ decrease in RR $(14.6 \% \pm 12.3 \%$ and $17.6 \%$ $\pm 13.5 \%$ vs $9.1 \% \pm 7.5 \%, P<0.05$ ), whereas no difference was observed between patients with mild OSA and non-OSA participants (Figure 2C).
In the healthy volunteers, the percentage decreases in RR and VT were $9.85 \% \pm 14.08 \%$ and $1.91 \% \pm 3.15 \%$, respectively, after two-breath pure oxygen inhalation (Supplementary Table 4). These results are consistent with the findings in a previous human study. ${ }^{16}$ Herein, we defined hCBC as a decrease of $>12 \%$ in RR after breathing of pure $\mathrm{O}_{2}$ (ie, a change percentage $20 \%$ greater than that observed in normal subjects). Accordingly, the prevalence of hCBC in the OSA group was $66.2 \%$, which was higher than that in the non-OSA group (21.1\%, $P<0.001$; Table 1). The percentage of patients with hCBC also increased with increasing OSA severity $(35.1 \%$, $72.5 \%$, and $80.0 \%$ for the mild, moderate, and severe OSA subgroups, respectively; Supplementary Table 1).

\section{Clinical Variables Associated with hCBC}

First, the associations between OSA features and $\mathrm{CBC}$ were investigated. Significant correlations were observed between $\mathrm{CBC}$ and AHI ( $\mathrm{r}=0.667, P<0.001)$, ODI $(\mathrm{r}=0.660$, $P<0.001)$, LAT $(\mathrm{r}=0.462, P<0.001)$, nadir $\mathrm{SpO}_{2}(\mathrm{r}=-0.370$, $P<0.001)$, mean $\mathrm{SpO}_{2}(\mathrm{r}=-0.371, P<0.001)$, and $\mathrm{F}_{\text {events-in- }}$ REM ( $\mathrm{r}=0.599, P=0.002$; Figure 3$)$. No correlations were observed between $\mathrm{CBC}$ and the other variables. As shown in Supplementary Table 2, stepwise multivariable linear regression analysis showed that $\mathrm{CBC}$ was associated with

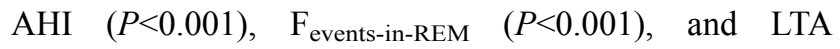
( $P=0.004$, adjusted $\left.\mathrm{r}^{2}=0.536, P=0.006\right)$. The other variables were excluded. 

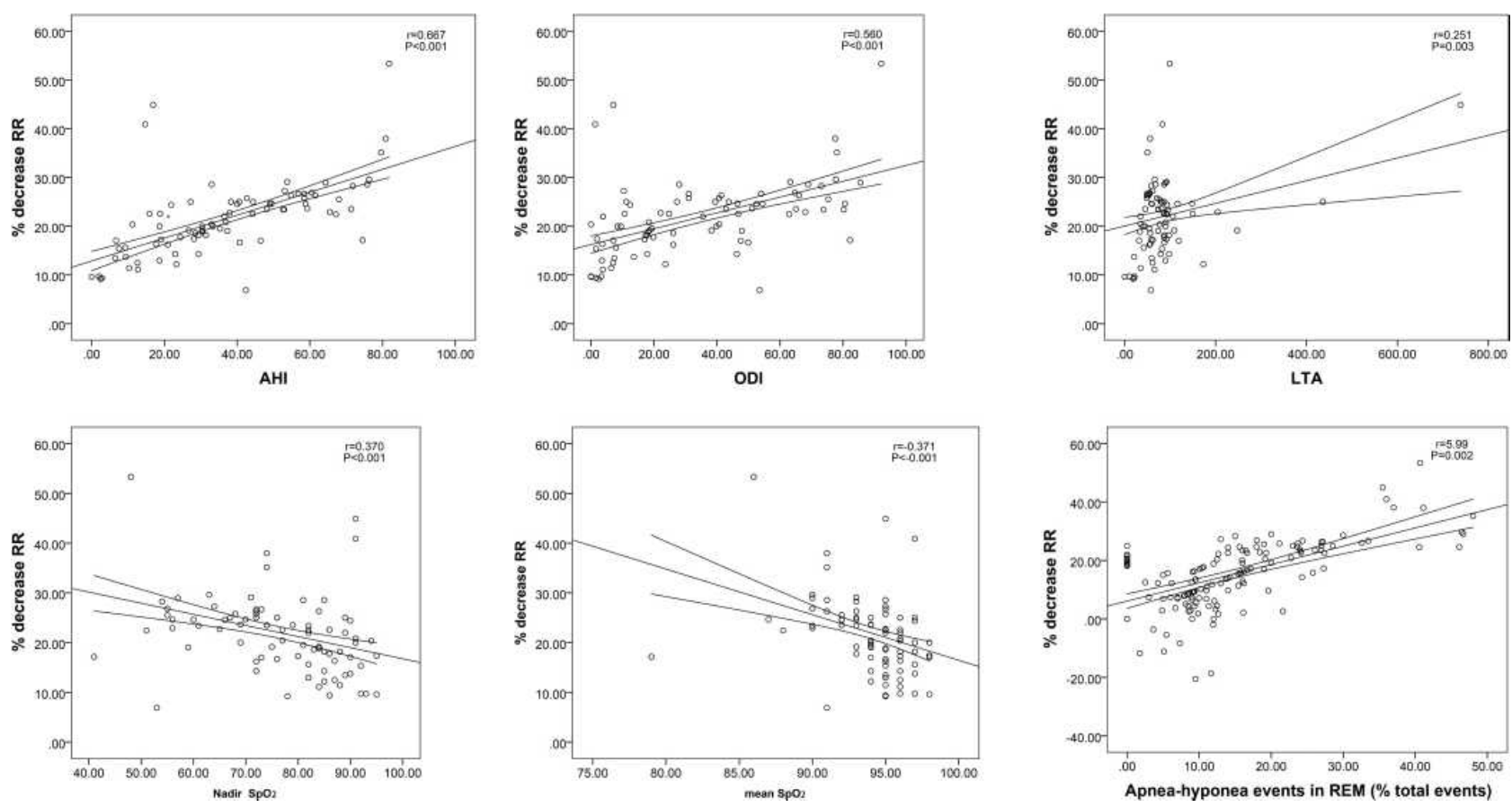

Figure 3 Correlation analysis of $\mathrm{CBC}$ (\% decrease in RR) with clinical variables among all study participants in the OSA and non-OSA groups. Strong correlations were found between CBC and AHI $(r=0.667, P<0.00 I)$, ODI $(r=0.660, P<0.001), F_{\text {events-in-REM }}(r=0.599, P=0.002)$, and LAT $(r=0.462, P<0.00 I) . N a d i r S_{p} O_{2}(r=-0.370, P<0.00 I)$ and mean $\mathrm{SpO}_{2}(r=-0.37 \mathrm{I}, \mathrm{P}<0.00 \mathrm{I})$ were weakly correlated with $\mathrm{CBC}$.

Abbreviations: AHI, apnea-hypopnea index; ODI, oxygen desaturation index; $\mathrm{SpO}_{2}$, pulse oxygen saturation; LTA, longest time of apnea.

Next, multivariable logistical regression analysis showed that AHI, LTA, and $\mathrm{F}_{\text {events-in-REM were significant indicators }}$ of $\mathrm{hCBC}(\mathrm{OR}=1.048,1.082$, and 1.024, respectively, all $P<0.05$; Table 2 and Supplementary Table 3). ROC curve analysis for each independent indicator was used to estimate the assessment validity for $\mathrm{hCBC}$ and yielded cutoff values of 15.7 events per hour for AHI, $48.4 \mathrm{~s}$ for LTA, and 12.7\% for $\mathrm{F}_{\text {events-in-REM }}$ (Supplementary Figure 1A-C). The

Table 2 Screening Model for Identifying High CB Chemosensitivity Developed by Multivariable Logistic Regression

\begin{tabular}{|l|l|l|l|}
\hline Variables & OR & $\mathbf{9 5 \%}$ CI & $P$ \\
\hline \multicolumn{4}{|l|}{ Multivariable logistic regression model - forward: conditional method } \\
\hline LTA & 1.024 & $1.004-1.045$ & 0.018 \\
AHI & 1.048 & $1.012-1.085$ & 0.009 \\
Fevents-in-REM $_{|l|}$ & 1.082 & $1.016-1.153$ & 0.014 \\
\hline Simplified multivariable logistic regression model with independent \\
binomial variables & 2.759 & $1.133-6.720$ & 0.025 \\
\hline LAT (>48.4 s) & 4.491 & $1.866-10.807$ & 0.001 \\
AHI (>15.7 events per hour) & 8.737 & $3.281-23.270$ & $<0.001$ \\
\hline
\end{tabular}

Abbreviations: AHI, apnea-hypopnea index; LTA, longest time of apnea; $F_{\text {events-in- }}$ REM, fraction of apnea-hypopnea events in rapid-eye-movement sleep (REM) (\% total events); $\mathrm{B}$, regression coefficient; $\mathrm{Cl}$, confidence interval. following simplified logistic regression model for $\mathrm{hCBC}$ with independent binomial variables was established: hCBC score $=1 \times($ LTA $>48.4 \mathrm{~s})+2 \times($ AHI $>15.7$ events per hour $)+3 \times\left(\mathrm{F}_{\text {events-in-REM }}>12.7 \%\right)$ (Figure 4$)$. This model allocated a score to each criterion according to its OR value, ie, 1 (LTA > 48.4 s), 2 (AHI > 15.7 events per hour), and 3 ( $\left.\mathrm{F}_{\text {events-in-REM }}>12.7 \%\right)$. The developed hCBC score criteria were validated by the scoring for each patient, and the results demonstrated the strong ability of the model to identify hCBC (area under the curve $[\mathrm{AUC}]=0.852$, 95\% confidence interval $[\mathrm{CI}]: 0.792-0.911, P<0.001$; Supplementary Figure 1D). With a cutoff value of 3 or greater, the developed screening model had a sensitivity of $79.4 \%$ and specificity of $88.2 \%$ for identifying hCBC.

\section{Discussion}

In this study, we observed that $\mathrm{CBC}$, determined by the Dejours test, was more pronounced in OSA patients than in individuals without OSA, which is consistent with the findings of Tafil-Klawe et al. ${ }^{21}$ In support of our hypothesis, $\mathrm{CBC}$ was associated with OSA severity. We further found that AHI, LTA, and $\mathrm{F}_{\text {events-in-REM were indepen- }}$ dently associated with hCBC. Accordingly, a screening model using AHI, LTA, and $\mathrm{F}_{\text {events-in-REM was developed }}$ and showed a good ability for identifying patients with 


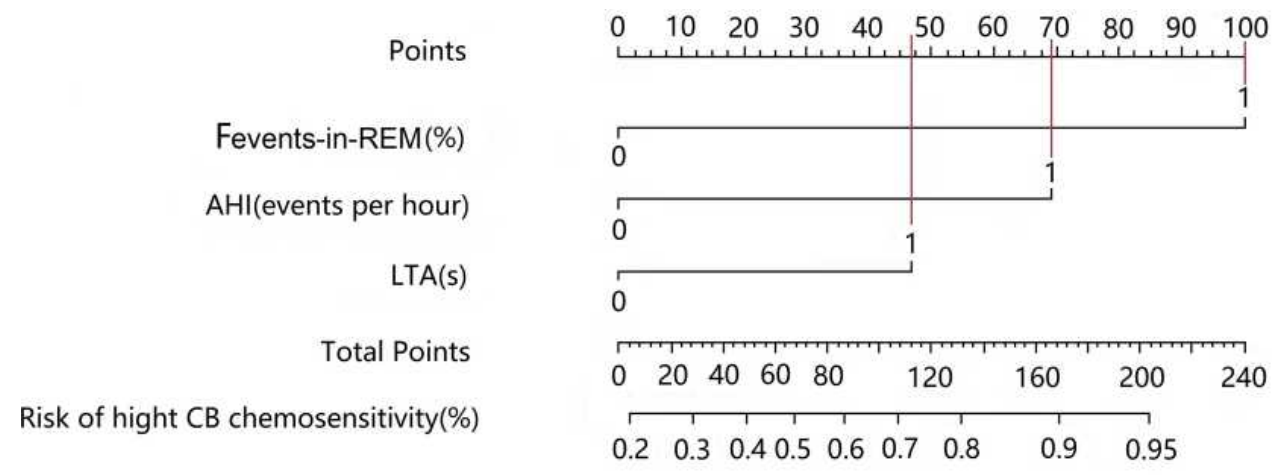

Figure 4 Nomogram of the logistic model including three independent binomial variables for identifying high carotid body chemosensitivity (hCBC). With this model, a vertical line is drawn from each variable upward to the points and then the corresponding point value is recorded (ie, " $F_{\text {events-in-REM }}=I$ " = I00 points). The point values for all variables are then summed to obtain a total score that corresponds to a probability of hCBC found at the bottom of the nomogram. The cutoff values were I5.7 events per hour for AHI, $48.4 \mathrm{~s}$ for LTA, and $12.7 \%$ for $\mathrm{F}_{\text {events-in-REM. }}$

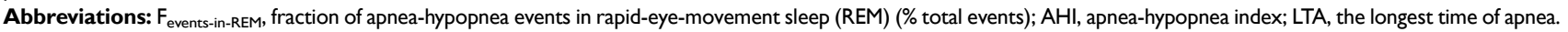

hCBC. Our results demonstrate that hCBC can be evaluated using a model integrating several variables from PSG.

The mechanisms underlying an increase in $\mathrm{CBC}$ in patients with OSA might involve the functional plasticity of the $\mathrm{CB}$, because the $\mathrm{CB}$ volume or the number of glomus cells were not modified when rats were exposed to intermittent hypoxia (IH) for 21 days. ${ }^{22}$ Specifically, only shortterm $\mathrm{IH}$, but not long-term $\mathrm{IH}$, resulted in selective augmentation of the hypoxic response of the $\mathrm{CB}$ and thus sensitized the $\mathrm{CB} .{ }^{23}$ Oxidative stress has been proposed as the key mediator of enhanced CBC induced by IH. IH increases the generation of reactive oxygen species (ROS) in the $\mathrm{CB},{ }^{24}$ and ROS signaling plays a vital role in mediating the sensory plasticity of the $\mathrm{CB} .{ }^{25}$ As shown in a rodent model, IH resulted in markedly elevated ROS levels and subsequent $\mathrm{H}_{2} \mathrm{~S}$ production in the $\mathrm{CB}$, and inhibition of $\mathrm{H}_{2} \mathrm{~S}$ synthesis in the $\mathrm{CB}$ could prevent IH-evoked sympathetic activation and hypertension. ${ }^{26}$

A consensus has yet to be reached regarding whether enhanced $\mathrm{CBC}$ is a causative factor or a consequent manifestation of OSA. On one hand, CBC is believed to be enhanced by $\mathrm{IH}^{27}$ Mechanically, $\mathrm{IH}$ was shown to markedly alter gaseous molecular signaling in the $\mathrm{CB}$, leading to changes in $\mathrm{CB}$ activity and possible enhancement of $\mathrm{CBC}^{26,28}$ On the other hand, some clinical studies have suggested that abnormal CB activity could be a driver of sleep apnea. ${ }^{6-8}$ Artificial alteration of gaseous molecular signaling in an animal model was found to also lead to sleep apnea via the induction of CB hyperactivity. ${ }^{29}$ In the present study, we found that only patients with severe OSA demonstrated significantly enhanced CBC compared to individuals without OSA, indicating that OSA exists before $\mathrm{CBC}$ enhancement given that $\mathrm{CB}$ hyperactivity occurred with the progression of OSA. However, these findings do not preclude the existence of a positive feedback loop between CBC enhancement and OSA progression, as $\mathrm{CB}$ hyperactivity itself could also trigger the exacerbation of OSA.

Until now, there have been no accurate epidemiological data for hCBC. In this study, $66.2 \%$ of OSA patients had hCBC based on our criteria for the results of the Dejours test (ie, percent decrease in RR 20\% greater than the normal value). hCBC leads to instability of respiratory central regulation, which is directly related to CSA, but also is involved in the pathogenesis of OSA. Furthermore, hCBC is associated with hypertension and insulin resistance. Therefore, it is of great practical significance to identify hCBC.

The Dejours test is obviously favorable with regard to safety and has been proven reproducible in specific populations. ${ }^{30}$ Increased CBC manifesting as a $\%$ decrease in RR is also seen in patients with chronic heart failure ${ }^{31}$ and prediabetes. ${ }^{32}$ Herein, CBC was evaluated using two breaths of $100 \% \mathrm{O}_{2}$, and this test can reveal a change in the oxygen drive for ventilation. ${ }^{16}$ The increased $\mathrm{CBC}$ observed in OSA patients was mainly seen in the change in $R R$ and not in a change in VT (Figure 2B and Table 1), consistent with the results obtained by Cunha-Guimaraes et al. ${ }^{32}$

In addition to Dejours test, the following indicators of autonomic nervous activity are used to evaluate $\mathrm{CBC}$ : muscle sympathetic nerve activity (MSNA) and heart rate variability (HRV). CB activation increases sympathetic activity and also simultaneously increases cardiac vagal as well as cardiac sympathetic nerve activity, 
causing HRV. ${ }^{33,34}$ OSA patients also have high sympathetic activity, even during normoxic wakefulness. ${ }^{35}$ HRV is a common quantitative index for evaluating sympatho-parasympathetic balance, ${ }^{36}$ which makes it a promising indicator of $\mathrm{CBC} .{ }^{37}$ However, the dose-effect relationships between $\mathrm{CBC}$ and MSNA as well as HRV have not been explored in the reported studies.

In the present study, we developed an assessment model for CBC using three parameters from PSG. AHI is a pivotal indicator of OSA severity. A high AHI enhances CB activity through ROS elevation mediated by $\mathrm{CIH}$ in OSA. A longer LTA may induce a longer stimulating time for $\mathrm{CB}$ during apnea and hypopnea and could lead to an increased CBC. CBC in mild OSA patients was not increased compared with that in individuals without OSA, which might partly be attributed to the lack of a significant increase in LTA with only mild OSA $(24.6 \pm 11.6$ vs $22.3 \pm 7.5 \mathrm{~s}, P=0.565)$.

REM sleep is associated with totally distinct physiological changes, including high sympathetic activity, lower vagal tone, and cardiovascular instability. ${ }^{13,14}$ Multiple neurotransmitters and their receptors are found in the $\mathrm{CB}$ (ie, norepinephrine, 5-hydroxytryptamine [5-HT], and dopamine) and can participate in CBC during REM sleep. ${ }^{38}$ The sympathetic fibers originating from the superior cervical ganglion innervate the $\mathrm{CB}$, and the stimulation of sympathetic fibers could modulate CBC via activation of specific adrenoceptor subtypes within the $\mathrm{CB}^{39}$ Thus, a higher $\mathrm{F}_{\text {events-in-REM might }}$ contribute to a stronger stimulation of the $\mathrm{CB}$. In addition, hCBC and sympathetic hyperfunction are also linked through IH in OSA. As mention before, IH induces $\mathrm{CB}$ alteration via ROS elevation. IH can also induce hypoxic sensitivity of the adrenal chromaffin cells due to increased ROS levels. ${ }^{24}$

The limitations of this study should be mentioned. First, the sample size is relatively small for a clinical study, but the results are statistically robust for a pilot study. Second, the relationship between $\mathrm{CBC}$ and respiratory control instability was not explored directly. Still our results can provide a direction for further studies of respiratory control instability and efforts to simplify the assessment of CBC.

In conclusion, hCBC is related to the severity of OSA. Our results demonstrated that $\mathrm{hCBC}$ can be evaluated using a scoring system based on clinical variables from PSG. Furthermore, the assessment model can be applied in further research to identify sub-phenotypes with hCBC and to guide individualized therapy for OSA.

\section{Acknowledgments}

The authors acknowledge the help of Mr. Guo Jun. This study was funded by the Key Research Program of Shanghai Science and Technology Commission (\#18140903600), the National Key Technology Research and Development Program of China (\#2018YFC1311900), and the Medical Research Project of Xuhui District (\#SHXH201926).

\section{Disclosure}

All authors declare that they have no competing interests.

\section{References}

1. Benjafield AV, Ayas NT, Eastwood PR, et al. Estimation of the global prevalence and burden of obstructive sleep apnoea: a literature-based analysis. Lancet Respir Med. 2019;7(8):687-698. doi:10.1016/ S2213-2600(19)30198-5

2. Kim J, Yoon DW, Lee SK, et al. Concurrent presence of inflammation and obstructive sleep apnea exacerbates the risk of metabolic syndrome: a KoGES 6-year follow-up study. Medicine (Baltimore). 2017;96(7):e4488. doi:10.1097/MD.0000000000004488

3. Torres G, Sanchez-de-la-torre M, Barbe F. Relationship Between OSA and Hypertension. Chest. 2015;148(3):824-832. doi:10.1378/chest.15-0136

4. Martinez-Garcia MA, Campos-Rodriguez F, Barbe F. Cancer and OSA: current evidence from human studies. Chest. 2016;150 (2):451-463. doi:10.1016/j.chest.2016.04.029

5. Eckert DJ. Phenotypic approaches to obstructive sleep apnoea - New pathways for targeted therapy. Sleep Med Rev. 2018;37:45-59. doi:10.1016/j.smrv.2016.12.003

6. Deacon-Diaz NL, Sands SA, Mcevoy RD, Catcheside PG. Daytime loop gain is elevated in obstructive sleep apnea but not reduced by CPAP treatment. J Appl Physiol (1985). 2018;125(5):1490-1497. doi:10.1152/japplphysiol.00175.2018

7. Edwards BA, Sands SA, Eckert DJ, et al. Acetazolamide improves loop gain but not the other physiological traits causing obstructive sleep apnoea. J Physiol. 2012;590(5):1199-1211. doi:10.1113/jphysiol.2011.223925

8. Wellman A, Malhotra A, Jordan AS, Stevenson KE, Gautam S, White DP. Effect of oxygen in obstructive sleep apnea: role of loop gain. Respir Physiol Neurobiol. 2008;162(2):144-151. doi:10.1016/j. resp.2008.05.019

9. Lam SY, Liu Y, Ng KM, et al. Chronic intermittent hypoxia induces local inflammation of the rat carotid body via functional upregulation of proinflammatory cytokine pathways. Histochem Cell Biol. 2012;137(3):303-317. doi:10.1007/s00418-011-0900-5

10. Smith CA, Blain GM, Henderson KS, Dempsey JA. Peripheral chemoreceptors determine the respiratory sensitivity of central chemoreceptors to CO2: role of carotid body CO2. J Physiol. 2015;593 (18):4225-4243. doi:10.1113/JP270114

11. Hess HW, Hostler D, Clemency BM, Johnson BD. Carotid body chemosensitivity at 1.6 ATA breathing air versus $100 \%$ oxygen. $J$ Appl Physiol (1985). 2020;129(2):247-256. doi:10.1152/ japplphysiol.00275.2020

12. Deacon-Diaz N, Malhotra A. Inherent vs. induced loop gain abnormalities in obstructive sleep apnea. Front Neurol. 2018;9:896. doi:10.3389/fneur.2018.00896

13. Narkiewicz K, van de Borne PJ, Pesek CA, Dyken ME, Montano N, Somers VK. Selective potentiation of peripheral chemoreflex sensitivity in obstructive sleep apnea. Circulation. 1999;99(9):1183-1189. doi:10.1161/01.cir.99.9.1183

14. Smith ML, Pacchia CF. Sleep apnoea and hypertension: role of chemoreflexes in humans. Exp Physiol. 2007;92(1):45-50. doi:10.1113/expphysiol.2006.033753 
15. Iturriaga R. Translating carotid body function into clinical medicine $J$ Physiol. 2018;596(15):3067-3077. doi:10.1113/JP275335

16. Dejours P. Chemoreflexes in breathing. Physiol Rev. 1962;42:335-358. doi:10.1152/physrev.1962.42.3.335

17. Dejours P. Control of respiration by arterial chemoreceptors. Ann N Y Acad Sci. 1963;109:682-695. doi:10.1111/j.1749-6632.1963. tb13497.x

18. Berry RB, Brooks R, Gamaldo CE; American Academy of Sleep Medicine, et al. The AASM Manual for the Scoring of Sleep and Associated Events: Rules, Terminology and Technical Specifications. Version 2.3. Darien, IL: American Academy of Sleep Medicine;2016.

19. Whelton PK, Carey RM, Aronow WS, et al. 2017 ACC/AHA/AAPA/ $\mathrm{ABC} / \mathrm{ACPM} / \mathrm{AGS} / \mathrm{APhA} / \mathrm{ASH} / \mathrm{ASPC} / \mathrm{NMA} / \mathrm{PCNA}$ Guideline for the prevention, detection, evaluation, and management of high blood pressure in adults: executive summary: a report of the american college of cardiology/American heart association task force on clinical practice guidelines. Hypertension. 2018;71(6):1269-1324. doi:10.1161/HYP.0000000000000066

20. Wesselink AK, Hatch EE, Rothman KJ, Mikkelsen EM, Aschengrau A, Wise LA. Prospective study of cigarette smoking and fecundability. Hum Reprod. 2019;34(3):558-567. doi:10.1093/ humrep/dey372

21. Tafil-Klawe M, Thiele AE, Raschke F, Mayer J, Peter JH, von Wichert W. Peripheral chemoreceptor reflex in obstructive sleep apnea patients; a relationship between ventilatory response to hypoxia and nocturnal bradycardia during apnea events. Pneumologie. 1991;45(Suppl 1):309-311.

22. Del RR, Munoz C, Arias P, Court FA, Moya EA, Iturriaga R. Chronic intermittent hypoxia-induced vascular enlargement and VEGF upregulation in the rat carotid body is not prevented by antioxidant treatment. Am J Physiol Lung Cell Mol Physiol. 2011;301(5):L702L711. doi:10.1152/ajplung.00128.2011

23. Peng YJ, Prabhakar NR. Effect of two paradigms of chronic intermittent hypoxia on carotid body sensory activity. J Appl Physiol (1985). 2004;96(3):1236-1242, 1196. doi:10.1152/japplphysiol.00820.2003

24. Nanduri J, Peng YJ, Yuan G, Kumar GK, Prabhakar NR. Hypoxiainducible factors and hypertension: lessons from sleep apnea syndrome. J Mol Med (Berl). 2015;93(5):473-480. doi:10.1007/ s00109-015-1274-2

25. Lucking EF, O'Connor KM, Strain CR, et al. Chronic intermittent hypoxia disrupts cardiorespiratory homeostasis and gut microbiota composition in adult male guinea-pigs. EBioMedicine. 2018;38:191-205. doi:10.1016/j.ebiom.2018.11.010

26. Prabhakar NR, Peng YJ, Yuan G, Nanduri J. Reactive oxygen radicals and gaseous transmitters in carotid body activation by intermittent hypoxia. Cell Tissue Res. 2018;372(2):427-431. doi:10.1007/ s00441-018-2807-0
27. Prabhakar NR, Peng YJ, Nanduri J. Hypoxia-inducible factors and obstructive sleep apnea. J Clin Invest. 2020;130(10):5042-5051. doi:10.1172/JCI137560

28. Peng YJ, Overholt JL, Kline D, Kumar GK, Prabhakar NR. Induction of sensory long-term facilitation in the carotid body by intermittent hypoxia: implications for recurrent apneas. Proc Natl Acad Sci U S A. 2003;100(17):10073-10078. doi:10.1073/pnas.1734109100

29. Peng YJ, Zhang X, Gridina A, et al. Complementary roles of gasotransmitters CO and H2S in sleep apnea. Proc Natl Acad Sci U S A. 2017;114(6):1413-1418. doi:10.1073/pnas.1620717114

30. Chardon K, Telliez F, Bach V, et al. Effects of warm and cool thermal conditions on ventilatory responses to hyperoxic test in neonates. Respir Physiol Neurobiol. 2004;140(2):145-153. doi:10.1016/j. resp.2003.11.007

31. Sinski M, Lewandowski J, Przybylski J, et al. Deactivation of carotid body chemoreceptors by hyperoxia decreases blood pressure in hypertensive patients. Hypertens Res. 2014;37(9):858-862. doi:10.1038/hr.2014.91

32. Cunha-Guimaraes JP, Guarino MP, Timoteo AT, et al. Carotid body chemosensitivity: early biomarker of dysmetabolism in humans. Eur J Endocrinol. 2020;182(6):549-557. doi:10.1530/EJE-19-0976

33. Sapru HN. Carotid chemoreflex. Neural pathways and transmitters. Adv Exp Med Biol. 1996;410:357-364.

34. Pachen M, Abukar Y, Shanks J, Lever N, Ramchandra R. Activation of the carotid body increases directly recorded cardiac sympathetic nerve activity and coronary blood flow in conscious sheep. Am $J$ Physiol Regul Integr Comp Physiol. 2020. doi:10.1152/ ajpregu.00246.2020

35. Somers VK, Dyken ME, Clary MP, Abboud FM. Sympathetic neural mechanisms in obstructive sleep apnea. J Clin Invest. 1995;96 (4):1897-1904. doi:10.1172/JCI118235

36. Kleiger RE, Stein PK, Bigger JJ. Heart rate variability: measurement and clinical utility. Ann Noninvasive Electrocardiol. 2005;10 (1):88-101. doi:10.1111/j.1542-474X.2005.10101.x

37. Rupprecht S, Hoyer D, Hagemann G, Witte OW, Schwab M. Central sleep apnea indicates autonomic dysfunction in asymptomatic carotid stenosis: a potential marker of cerebrovascular and cardiovascular risk. Sleep. 2010;33(3):327-333. doi:10.1093/sleep/33.3.327

38. Kumar P, Prabhakar NR. Peripheral chemoreceptors: function and plasticity of the carotid body. Compr Physiol. 2012;2(1):141-219. doi:10.1002/cphy.c100069

39. Almaraz L, Perez-Garcia MT, Gomez-Nino A, Gonzalez C. Mechanisms of alpha2-adrenoceptor-mediated inhibition in rabbit carotid body. Am J Physiol. 1997;272(2 Pt 1):C628-C637. doi:10.1152/ajpcell.1997.272.2.C628

\section{Publish your work in this journal}

Nature and Science of Sleep is an international, peer-reviewed, open access journal covering all aspects of sleep science and sleep medicine, including the neurophysiology and functions of sleep, the genetics of sleep, sleep and society, biological rhythms, dreaming, sleep disorders and therapy, and strategies to optimize healthy sleep.
The manuscript management system is completely online and includes a very quick and fair peer-review system, which is all easy to use. Visit http://www.dovepress.com/testimonials.php to read real quotes from published authors. 